held to brainstorm ideas and to develop training materials. A WhatsApp group was initiated to maintain continuous links within this team. The four pillars of FiCare were addressed: Staff Education, Parent Education, NICU Environment and Psycho-Social Support.

After months of preparation and staff training, FiCare was launched at the end of October 2016. Step by step over the following months we implemented further aspects of our FiCare model. FiCare is established as standard care for all admissions to our NICU.

Results Badgernet was used to obtain outcome measures in two epochs: 1 st December 2015 - 1 st October 2016 (PreFiCare) and 1 st December 2016 - 1 st October 2017 (PostFiCare).

\begin{tabular}{lll} 
Abstract G213(P) Table 1 & & \\
\hline Outcome & $\begin{array}{l}\text { Pre-ficare } \\
\text { epoch }\end{array}$ & $\begin{array}{l}\text { Post-ficare } \\
\text { epoch }\end{array}$ \\
\hline NICU days receiving any of own mother's milk & $76 \%$ & $81 \%$ \\
NICU days receiving only own mother's milk & $56 \%$ & $63 \%$ \\
Babies born<33 weeks gestation discharged receiving any & $66 \%$ & $80 \%$ \\
of own mother's milk & & \\
Length of stay>90 th centile for UK, based on gestation & $6.3 \%$ & $3.9 \%$ \\
\hline
\end{tabular}

The results in table 1 also compare very favourably with our network, where to date, ours is the only unit to implement FiCare.

Conclusion Since FiCare launched, there has been an improvement in breast feeding rates and a reduction in length of stay. We have also noted a gradual cultural change on NICU, including increased awareness of developmentally appropriate practices and increased parents' readiness for discharge.

\section{G214(P) PRETERM INFANT TRANSFUSION SUPPORT: ADULT OR CORD BLOOD DONATION? A LONGITUDINAL STUDY OF CD71 EXPRESSION IN PRETERM, CORD AND ADULT BLOOD SAMPLES}

${ }^{1} \mathrm{M}$ Forrester, ${ }^{2} \mathrm{~S}$ Mohamed Cassim, ${ }^{3} \mathrm{~S}$ Armstrong-Fisher, ${ }^{1,3} \mathrm{M}$ Vickers. ${ }^{1} / \mathrm{mm}$ munology, University of Aberdeen, Aberdeen, UK; ${ }^{2}$ Neonatology, NHS Grampian, Aberdeen, UK; ${ }^{3}$ Blood Transfusion, Scottish National Blood Transfusion Service, Aberdeen, UK

\subsection{6/archdischild-2018-rcpch.209}

Background Several risk factors are implicated in the development of necrotizing enterocolitis (NEC), one of which is receiving a blood transfusion. Recent evidence suggests that newborn (cord) red blood cells expressing CD71 can suppress potentially damaging immune responses to bacteria colonising the gut. Therefore, a blood transfusion, currently from adult donors, might effectively dilute these preventative properties, resulting in damaging inflammatory responses against gut bacteria, and subsequent NEC.

Aim We wished to identify in neonatal cord blood samples the early red cell population $(\mathrm{CD} 71+/ \mathrm{CD} 235+)$ purportedly responsible for the immunosuppression, and to establish a time course of their development with respect to gestational age. We also compared to adult blood donors, and assessed their potential anti-inflammatory activity.
Method Residual routine blood samples were obtained from preterm babies in the Neonatal Unit, after informed parental consent. Sequential samples were obtained until the infants were discharged. Neonatal/cord/adult blood samples, were separated by density centrifugation into mononuclear (buffy) cell layer (MNC) and red cells (RBC). MNC and RBC populations were immunofluorescently labelled with anti-human CD71 and anti-human CD235a and analysed by flow cytometry. We have assessed normal umbilical cord (term, elective sections) and healthy adult blood to investigate the anti-inflammatory properties of newborn cord RBC compared to adult RBC in simulated in vitro transfusions. Pro- and anti-inflammatory cytokine production by innate immune cells in response to pathogenic gut bacteria were also evaluated.

Results The proportion of RBC and MNC CD71 + cells were significantly greater $(\mathrm{p}<0.0001)$ from neonates (term cord and preterm infants) compared to adults. While transfusion did elicit suppression of pro-inflammatory cytokine production in response to bacterial challenge, regardless of the donor $\mathrm{RBC}$ source, the net effect of adult RBC transfusion is dilution of the innate CD71 + population.

Conclusion Logistics aside, we suggest that transfusion using umbilical cord progenitor $\mathrm{RBC}$, rather than adult $\mathrm{RBC}$ donations, might afford an alternative option for pre-term infants to lessen their risk of post-transfusion associated NEC.

\section{G215(P) IMPROVING SAFETY AT THE VERY BEGINNING: A NOVEL NEONATAL DELIVERY SAFETY CHECKLIST}

AM Taylor, P Lewis, M Nugent, J Montague, S Mitra. Neonatal Unit, University College London Hospitals NHS Foundation Trust, London, UK

\subsection{6/archdischild-2018-rcpch.210}

Aim Resuscitation and stabilisation of newborn babies can be a complex process involving members of several multi-disciplinary teams. We aimed to improve early newborn care by reducing errors and improving safety at each stage of this process. The World Health Organisation (WHO) Surgical Safety Checklist ${ }^{1,2}$ has been shown to dramatically reduce morbidity and mortality in surgical patients. Using the same principles, we created a novel Neonatal Delivery Safety Checklist with an aim to improve multi-disciplinary communication and early care of the newborn.

Method We started by surveying all members of the multi-disciplinary team about their experiences of attending complex and preterm deliveries. We process mapped the stages involved in attending a delivery and combined this with the survey information. This formed the basis of the pilot project where, as in the WHO Surgical Safety Checklist, we identified three 'vital phases': a preparatory period; a pause before the delivery where the whole team is encouraged to answer the question 'are we ready?'; and before transfer to the neonatal unit. Results We piloted the checklist over two weeks and immediate feedback was gathered. A 'plan, do, study, act' cycle was then initiated to respond to feedback from the multi-disciplinary team. As a result, the Checklist has gone through seven variations to date to make it as user friendly as possible whilst retaining the key principles.

Admission temperature in babies born at $<32$ weeks has shown a dramatic improvement since The Checklist has been introduced for routine preterm deliveries. Nearly $50 \%$ of infants had admission temperature $<36.5^{\circ} \mathrm{C}$ in the month 